\title{
Short communication: ELISA system for screening of bovine mastitis caused by Prototheca zopfii
}

\author{
Rui Kano, ${ }^{* 1}$ Ayano Sato, $†$ Hideto Sobukawa, ${ }^{*}$ Yuko Sato, ${ }^{*}$ Takaaki Ito, $\ddagger$ Kazuyuki Suzuki, $\dagger$ \\ Atsuhiko Hasegawa,§ and Hiroshi Kamata* \\ *Department of Pathobiology, Nihon University School of Veterinary Medicine, 1866 Kameino, Fujisawa, Kanagawa 252-8510, Japan \\ †Department of Large Animal Clinical Sciences, School of Veterinary Medicine, Rakuno Gakuen University, 582 Midorimati, Bunnkyoudai, Ebetsu, \\ Hokkaido 069-8501, Japan \\ $\ddagger$ Okazaki Branch of Veterinary Hospital Aichi, Prefectural Federation of Agricultural Mutual Aid Associations (PFAMAA), 91-1, Oike, Hane-cho, \\ Okazaki, Aichi 444-0816, Japan \\ §Teikyo University Institute of Medical Mycology, 539 Otsuka, Hachioji, Tokyo 192-0395, Japan
}

\begin{abstract}
Prototheca zopfii is an achlorophyllic alga that causes bovine mastitis, resulting in a reduction in milk production and the secretion of thin, watery milk with white flakes. This study evaluated the use of an ELISA system for distinguishing cows with mastitis due to P. zopfii genotype 2 from healthy cows and cows with chronic candidal mastitis. We also investigated the transitional changes of specific antibody titers in healthy cows injected with inactivated $P$. zopfii genotype 2 cells. The ELISA system exhibited the highest sensitivity (94\%) and specificity (100\%) for chronic protothecal mastitis when the positive cutoff value was set at 43.4 ELISA units. Anti-protothecal IgG titers were positive in all cows after they were inoculated with inactivated $P$. zopfii genotype 2 cells. These results indicated that ELISA detection of anti-protothecal IgG in serum provided specificity and sensitivity sufficient for diagnosing protothecal mastitis. Thus, an ELISA system incorporating this specific antiserum is expected to be valuable for definitive field-based diagnosis of bovine mastitis due to $P$. zopfii genotype 2 .
\end{abstract}

Key words: bovine Prototheca mastitis, ELISA, diagnosis, immunization

\section{Short Communication}

Prototheca are achlorophyllic algae that are ubiquitous in cow-barn environments. Prototheca zopfii is associated with bovine mastitis, causing secretion of thin, watery milk with white flakes and reducing milk production. Prototheca zopfii has been biochemically and serologically identified as having 2 genotypes: genotype 1 and genotype 2 (Roesler et al., 2003, 2006).

Received March 14, 2016.

Accepted May 1, 2016.

${ }^{1}$ Corresponding author: kanou.rui@nihon-u.ac.jp
Virtually all isolates from bovine mastitis in Germany, Italy, Japan, Portugal, and Poland have been identified as $P$. zopfii genotype 2, suggesting that this genotype is the main causative agent in bovine protothecal mastitis (Roesler et al., 2003, 2006; Möller et al., 2007; Marques et al., 2008; Osumi et al., 2008; Kishimoto et al., 2010; Ricchi et al., 2010; Jagielski et al., 2011). Most cases of bovine protothecal mastitis in Japan are chronic and subclinical. Typically, the identification of $P$. zopfii as the causative agent in mastitis depends on a positive result from culture tests or analysis of milk samples (Roesler et al., 2003, 2006; Osumi et al., 2008). However, culturing and molecular analysis are not performed in all clinical cases. As a result, $P$. zopfii has been frequently misidentified under the microscope as Candida or another yeast. Assays that incorporate antibodies with specificity for $P$. zopfii genotype 2 could facilitate the definitive diagnosis of this infection.

Only a small number of studies have reported the use of ELISA for the diagnosis of mastitis (Roesler et al., 2001; Roesler and Hensel, 2003). An ELISA based on the detection of anti-protothecal $\operatorname{IgG}$ in serum has shown reliable sensitivity and specificity in distinguishing cows with chronic protothecal mastitis from their healthy counterparts (Roesler et al., 2001; Roesler and Hensel, 2003). However, to our knowledge, ELISA has not been investigated or evaluated for distinguishing between Prototheca and Candida in cases of bovine mastitis.

We developed and evaluated an ELISA system for detecting cows with protothecal mastitis and animals inoculated with $P$. zopfii genotype 2 cells. All procedures were carried out in accordance with the Guide for the Care and Use of Laboratory Animals of Rakuno Gakuen University and the Guide for the Care and Use of Laboratory Animals of the Japanese Ministry of Education, Culture, Sports, Science, and Technology.

Thirty-five serum samples were collected from 3 groups as follows: the first consisted of 16 cows with 
chronic mastitis from which $P$. zopfii genotype 2 had been cultured; the second consisted of 4 cows with chronic candidal mastitis from which P. zopfii had not been cultured; and the third consisted of 15 healthy cows. No other pathological agents were recovered from the cows. Culturing and identification of $P$. zopfii genotype 2 were performed as described in a previous report (Osumi et al., 2008).

Antigen preparation and ELISA procedures were carried out as described in previous reports (Roesler et al., 2001; Roesler and Hensel, 2003) with minor modifications. In brief, cells of P. zopfii genotype 2 SAG $2021^{\mathrm{T}}$ (Roesler et al., 2006) were incubated for $48 \mathrm{~h}$ at $37^{\circ} \mathrm{C}$ in liquid Prototheca isolation medium (Pore, 1973). After pelleting by centrifugation, algae were suspended in $0.05 \mathrm{M}$ of carbonate buffer $(\mathrm{pH} 9.6)$ and the density was adjusted to $10^{7}$ cells $/ \mathrm{mL}$. The cells were then sonicated at $4^{\circ} \mathrm{C}$. The concentration of the resulting protein suspension was adjusted to $10 \mu \mathrm{g} / \mathrm{mL}$ in carbonate buffer. (The minor modification was sonication for cells at $4^{\circ} \mathrm{C}$ to assay the proteins.) For the coating of wells, the suspension was distributed at $50 \mu \mathrm{L}$ per well into 96-well microtiter plates (Thermo Scientific, Roskilde, Denmark) and the plates were incubated at $37^{\circ} \mathrm{C}$ for $1 \mathrm{~h}$. Samples of each of the 35 serum samples (for the 3 groups described above) were distributed to separate precoated wells and incubated at $37^{\circ} \mathrm{C}$ for $1 \mathrm{~h}$. Antibody-antigen complexes were labeled using horseradish peroxidase-conjugated polyclonal anti-bovine IgG (Biorbyt, Berkeley, CA) as the secondary antibody, and binding was detected using standard colorimetric assay reagents.

The ELISA data were calculated using a computerbased program developed for ELISA evaluation (Roesler et al., 2001; Roesler and Hensel, 2003). Relative values of antibody activities in the positive standards were set at 100 ELISA units. The cutoff ELISA unit value was defined as 3 standard deviations above the mean calculated for the values obtained in all 3 groups. Significant differences in ELISA unit data between Prototheca-positive (16 protothecal serum samples) and Prototheca-negative groups (15 healthy cows and 4 chronic candidal mastitis serum samples) were checked by Student's $t$-test for unpaired observations or by Welch's test (Excel 2010; Microsoft, Tokyo, Japan). A $P$-value of $\leq 0.05$ was considered significant. The ELISA results were evaluated by calculating sensitivity and specificity.

For use in immunization, cells of $P$. zopfii genotype $2 \mathrm{SAG} 2021^{\mathrm{T}}$ were incubated for $72 \mathrm{~h}$ at $37^{\circ} \mathrm{C}$ in liquid Prototheca isolation medium (Pore, 1973). Algae were centrifuged and inactivated (fixed) in $0.05 \%$ formamide in $\mathrm{PBS}$ at $4^{\circ} \mathrm{C}$ overnight and then centrifuged and washed 2 times in physiological saline. An aliquot of inactivated cells ( $1 \mathrm{~mL}, 10^{8}$ cells) was combined with an equal volume of Freund's incomplete adjuvant (MP Biomedicals, Osaka, Japan) and mixed well.

The resulting antigen suspension (inactivated Prototheca cells) was injected intramuscularly at $2 \mathrm{~mL} /$ animal into the femur of 10 healthy 1-yr-old Holstein cows. After $4 \mathrm{wk}$, the injection was repeated. Physical examination at the injection sites detected no side effects in any of the inoculated cows. The inoculation protocol was based information from the Japanese Society for Animal Vaccine and Biomedical Research (http://www.jsavbr.jp/index.php).

Serum samples were collected every 2 wk starting 2 wk after initial inoculation. Evaluation of the ELISA in the 3 groups is shown in Figure 1. The positive cutoff value was defined as a value 3 standard deviations above the mean ELISA unit value of the 3 groups (Figure 1). A significant discrimination between Prototheca positive and negative (Candida-infected and healthy) cows $(P<0.001)$ was confirmed when serum IgG levels were measured (Figure 1A). The results of the ELISA indicated that the highest sensitivity was $94 \%$, specificity was $100 \%$, and the positive cutoff value was 43.4 ELISA units (Figure 1B).

At the first time point ( $2 \mathrm{wk}$ after initial immunization), anti-protothecal IgG titers in all experimental cows were below the positive cutoff value. Titers subsequently increased, exceeding the cutoff value after the second (booster) inoculation (Figure 2). The titer remained positive for $8 \mathrm{wk}$ in 9 of the 10 experimental animals (Figure 2).

This study consisted of 2 parts. In the first part, we evaluated the use of an ELISA system for distinguishing between cows with mastitis due to P. zopfii genotype 2 and healthy cows or cows with chronic mastitis due to Candida. In the second part, we investigated changes in the protothecal-specific antibody titers of healthy cows following inoculation with inactivated $P$. zopfii genotype 2 cells.

Anti-protothecal IgG titers were positive in all cows following antigen exposure. In a preliminary study, 1 $\mathrm{mL}$ of $10^{7}$ inactivated cells and an equal volume of the adjuvant did not induce enough positive antigen titers in cows (data not shown).

These results indicate that the ELISA detection system for anti-protothecal IgG in serum was specific and sensitive enough to permit diagnosis of protothecal mastitis. Our results were consistent with those of Roesler and Hensel (2003), who reported that ELISA for the detection of IgG in serum could be used for identification in nonlactating cows or cows with atretic udder quarters. 

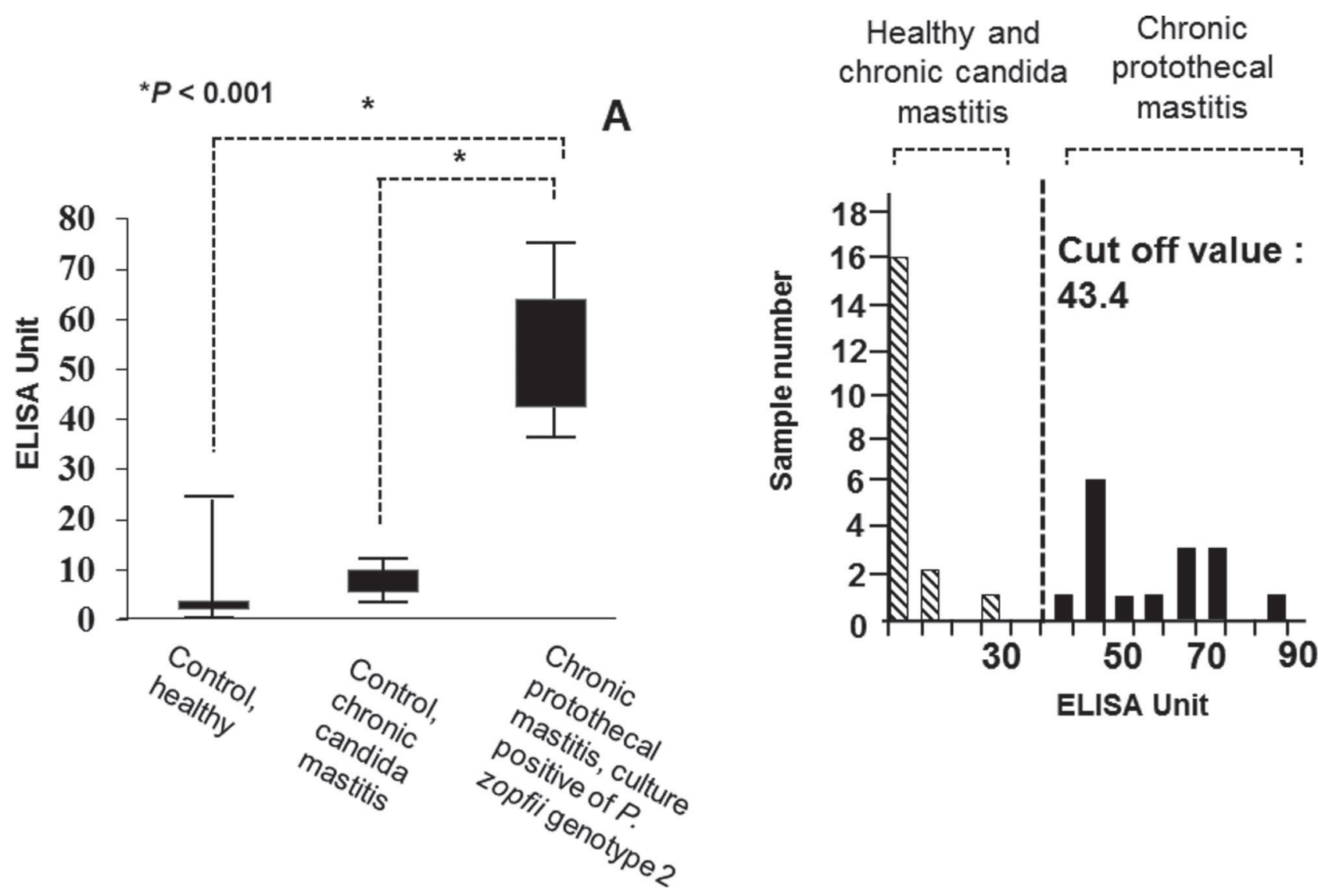

Figure 1. The ELISA activity of anti-Prototheca IgG in serum (A) and definition of the positive cutoff value (B). Asterisks indicate a significant discrimination value for distinguishing between Prototheca culture-positive cows and cows with candidal mastitis or healthy cows $(P<$ 0.001). The dotted vertical line indicates the positive cutoff value. Error bars indicate minimum and maximum of ELISA units.

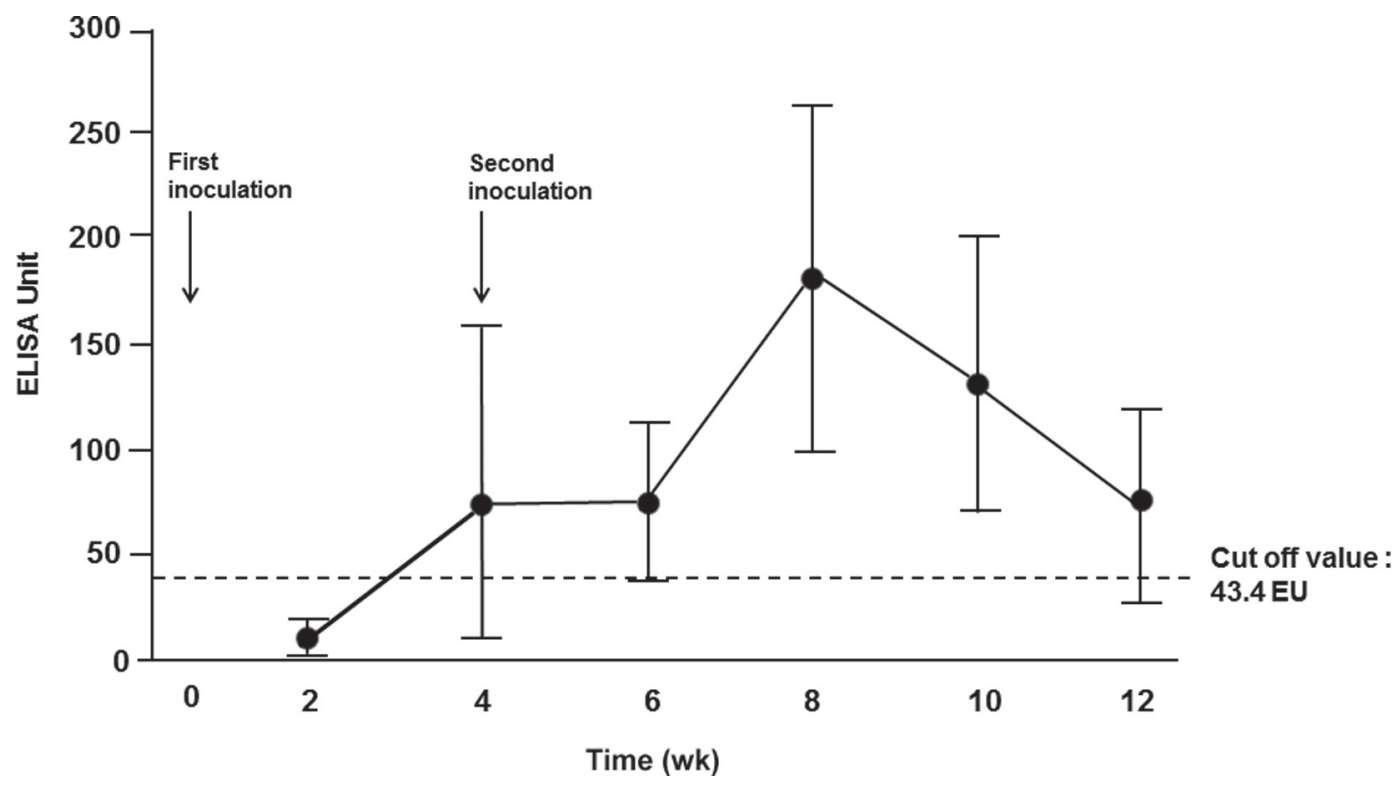

Figure 2. Anti-Prototheca IgG titers in antigen-inoculated cows. Inactivated Prototheca zopfii genotype $2 \mathrm{SAG}_{2021^{\mathrm{T}}}\left(10^{8}\right.$ cells $/$ dose) were injected into 10 healthy cows at 0 and 4 wk. Serum samples were collected every 2 wk starting 2 wk after initial inoculation. The dotted horizontal line indicates the positive cutoff value. Black circles indicate averages of antibody titers in antigen-inoculated cows. Error bars indicate standard deviations of antigen titers. 
Roesler and Hensel (2003) observed that animals testing positive for serum Prototheca-specific antibodies were intermittent shedders of the algae. In that study, approximately $21 \%$ of shedders showed a specific antibody response 6 mo before they first tested positive by culture. Therefore, ELISA-based systems may detect cows that are culture-positive or intermittently shedding. However, ELISA-based systems were evaluated by the same authors, and only a few papers have described a serologic approach aimed at detecting Prototheca-infected cows (Roesler et al., 2001; Roesler and Hensel, 2003). In the present study, ELISA procedures were carried out as described in previous reports (Roesler et al., 2001; Roesler and Hensel, 2003), with minor modifications. Our aim was to reevaluate the ELISA system using Japanese bovine protothecal mastitis cases, Candida mastitis cases, and Protothecainoculated cows, and we confirmed that ELISA systems incorporating Prototheca-specific antiserum may be of use for the definitive field diagnosis of bovine mastitis due to $P$. zopfii genotype 2 .

\section{ACKNOWLEDGMENTS}

This study was supported by Grants-in-Aid for Scientific Research (C) (22580368) from the Academic Frontier Project of the Japanese Ministry of Education, Culture, Sports, Science and Technology (MEXT), and from Nihon University (Kanagawa, Japan). The authors report no conflicts of interest. The authors alone were responsible for the content and writing of the paper.

\section{REFERENCES}

Jagielski, T., H. Lassa, J. Ahrholdt, E. Malinowski, and U. Roesler. 2011. Genotyping of bovine Prototheca mastitis isolates from Poland. Vet. Microbiol. 149:283-287.

Kishimoto, Y., R. Kano, H. Maruyama, M. Onozaki, K. Makimura, T. Ito, K. Matsubara, A. Hasegawa, and H. Kamata. 2010. 26S rDNA-based phylogenetic investigation of Japanese cattle-associated Prototheca zopfii isolates. J. Vet. Med. Sci. 72:123-126.

Marques, S., E. Silva, C. Kraft, J. Carvalheira, A. Videira, V. A. Huss, and G. Thompson. 2008. Bovine mastitis associated with Prototheca blaschkeae. J. Clin. Microbiol. 46:1941-1945.

Möller, A., U. Truyen, and U. Roesler. 2007. Prototheca zopfii genotype 2-The causative agent of bovine protothecal mastitis? Vet. Microbiol. 120:370-374.

Osumi, T., Y. Kishimoto, R. Kano, M. Maruyama, M. Onozaki, K. Makimura, T. Ito, K. Matsubara, and A. Hasegawa. 2008. Prototheca zopfii genotypes isolated from cow barns and bovine mastitis in Japan. Vet. Microbiol. 131:419-423.

Pore, R. S. 1973. Selective medium for the isolation of Prototheca. Appl. Microbiol. 26:648-649.

Ricchi, M., M. Goretti, E. Branda, G. Cammi, C. A. Garbarino, B. Turchetti, P. Moroni, N. Arrigoni, and P. Buzzini. 2010. Molecular characterization of Prototheca strains isolated from Italian dairy herds. J. Dairy Sci. 93:4625-4631.

Roesler, U., and A. Hensel. 2003. Longitudinal analysis of Protothece zopfii-specific immune responses: Correlation with disease progression and carriage in dairy cows. J. Clin. Microbiol. 41:1181-1186.

Roesler, U., A. Möller, A. Hensel, D. Baumann, and U. Truyen. 2006. Diversity within the current algal species Prototheca zopfii: A proposal for two Prototheca zopfii genotypes and description of a novel species, Prototheca blaschkeae sp. nov. Int. J. Syst. Evol. Microbiol. 56:1419-1425.

Roesler, U., H. Scholz, and A. Hensel. 2001. Immunodiagnostic identification of dairy cows infected with Prototheca zopfii at various clinical stages and discrimination between infected and uninfected cows. J. Clin. Microbiol. 39:539-543.

Roesler, U., H. Scholz, and A. Hensel. 2003. Emended phenotypic characterization of Prototheca zopfii: A proposal for three biotypes and standards for their identification. Int. J. Syst. Evol. Microbiol. 53:1195-1199. 